\title{
Validez y confiabilidad del cuestionario Orientación ante la Vida de Antonovsky (SC-29 ítems), versión en español*
}

\author{
Validity and reliability of Antonovsky's Orientation to Life questionnaire (SC-29 items), Spanish version \\ Validade e confiabilidade do Questionário Orientação para Viver de Antonovsky (SC-29 itens), versão em espanhol
}

Natalia Esquivel Garzón ${ }^{\mathrm{a}}$

Universidad Nacional de Colombia, Colombia

DOI: https://doi.org/10.11144/Javeriana.ie22.vcco nesquivelg@unal.edu.co

ORCID: https://orcid.org/0000-0002-5354-6774

Recibido: 07 Julio 2019

Luz Patricia Diaz Heredia Publicado: 22 Julio 2020

Universidad Nacional de Colombia, Colombia
ORCID: https://orcid.org/0000-0002-282X

\section{Resumen:}

Introducción: El cuestionario Orientación ante la Vida mide el sentido de coherencia, un concepto relacionado con el uso de los recursos de afrontamiento que influyen positivamente en una mejor calidad de vida y bienestar de aquellos adultos con enfermedad cardiovascular. Objetivo: Determinar la validez y confiabilidad del cuestionario Orientación ante la Vida de Antonovsky, en adultos con hipertensión arterial. Método: Estudio de tipo metodológico en el que se realizó la adaptación cultural y se determinó la validez facial, de contenido, de constructo y confiabilidad. En la adaptación cultural se siguió un proceso de traducción, consenso y retrotraducción. La validez facial y de contenido se cumplió mediante el juicio de expertos, utilizando las pruebas estadísticas de coeficiente kappa de Fleiss e índice de Lawshe modificado. En la validez de constructo y prueba de confiabilidad participaron 220 personas. La confiabilidad se calculó mediante la prueba estadística de alfa de Cronbach. Resultados: En la validez facial, el instrumento reportó un índice kappa de Fleiss mayor a 0,41, interpretado como un acuerdo moderado. El índice de validez de contenido fue satisfactorio con 0,87 , el análisis factorial exploratorio reportó tres factores con una varianza total explicada del 36,21 \%. El alfa de Cronbach fue de 0,82 para la escala total. Conclusiones: La versión en español del instrumento Orientación ante la Vida presenta un adecuado índice de validez de contenido y confiabilidad para uso en el contexto local. Es necesario continuar su aplicación en otros grupos y momentos para continuar refinando su validez.

Palabras clave: psicometría, estudios de validación, sentido de coherencia, enfermería.

\section{Abstract:}

Introduction: The Orientation to Life questionnaire measures the sense of coherence, a concept related to the use of coping resources that have a positive influence leading to a better quality of life and sense of well-being for adults with cardiovascular disease. Objective: To determine the validity and reliability of Antonovsky's Orientation to Life questionnaire in adults with arterial hypertension. Method: Methodological study in which cultural adaptation was carried out and the reliability and face, content, and construct validity were determined. In the cultural adaptation, a process of translation, consensus, and back-translation was followed. Content and face validity were met by expert judgment, using the Fleiss' kappa coefficient and modified Lawshe index statistical tests. Two hundred and twenty people participated in the construct validity and reliability test. Reliability was calculated using the Cronbach's alpha statistical test. Results: In terms of face validity, the instrument reported a Fleiss kappa index greater than 0.41 , interpreted as moderate agreement. The content validity index was satisfactory with 0.87 ; the exploratory factor analysis reported three factors with a total explained variance of $36.21 \%$. Cronbach's alpha was 0.82 for the full scale. Conclusions: The Spanish version of the Orientation to Life instrument presents an adequate content validity and reliability index for use in the local context. It is necessary to continue its application in other groups and times to continue refining its validity.

Keywords: psychometry, validation studies, sense of coherence, nursing.

\section{Resumo:}

Introdução: O questionário Orientação para Viver mede o senso de coerência, um conceito relacionado ao uso dos recursos de enfrentamento que influenciam positivamente uma melhor qualidade de vida e bem-estar de adultos com doença cardiovascular. Objetivo: Determinar a validade e confiabilidade do questionário Orientação para Viver de Antonovsky, em adultos com

Notas de autor

\footnotetext{
a Autora de correspondencia. Correo electrónico: nesquivelg@unal.edu.co
} 
hipertensão arterial. Método: Estudo de tipo metodológico em que se realizou adaptação cultural e determinou-se a validade facial, de conteúdo, de constructo e confiabilidade. $\mathrm{Na}$ adaptação cultural foi seguido um processo de tradução, consenso e retrotradução. A validade facial e de conteúdo foram atendidas por julgamento de especialistas, utilizando testes estatísticos de coeficiente kappa de Fleiss e índice de Lawshe modificado. Na validade de constructo e teste de confiabilidade participaram 220 pessoas. A confiabilidade foi calculada usando o teste estatístico de alfa de Cronbach. Resultados: Na validade facial, o instrumento relatou um índice kappa de Fleiss maior a 0,41 , interpretado como uma concordância moderada. O índice de validade de conteúdo foi satisfatório com 0,87 , a análise fatorial exploratória mostrou três fatores com variância total explicada de 36,21\%. O alfa de Cronbach foi de 0,82 para a escala total. Conclusões: A versão em espanhol do instrumento Orientação para Viver apresenta adequado índice de validade de conteúdo e confiabilidade para uso no contexto local. É preciso continuar a sua aplicação em outros grupos e momentos para continuar refinando sua validade.

Palavras-chave: psicometria, estudos de validação, senso de coerência, enfermagem.

\section{Introducción}

La hipertensión arterial (HTA) se considera una enfermedad crónica no transmisible, que es el principal factor de riesgo independiente para el desarrollo de enfermedad cardiovascular, accidente cerebrovascular e insuficiencia renal (1), además de ser un problema de salud pública en los países de ingresos bajos y medios, ya que afecta aproximadamente a un tercio de su población (2). Para el adecuado control de la enfermedad, es importante que el paciente realice cambios en el estilo de vida y se adhiera al tratamiento, lo cual a su vez implica adaptarse a la nueva condición de salud (3); usar capacidades, recursos personales y estrategias de afrontamiento para tener control sobre la propia salud, y llevar una vida saludable a pesar de la presencia de la enfermedad (4).

El sentido de coherencia (SC) es un concepto central de la teoría salutogénica propuesta por el sociólogo Aaron Antonovsky, en respuesta a la búsqueda de los factores que contribuyen a mantener la salud física y el bienestar psicológico (5); corresponde a un recurso personal conformado por tres dimensiones: 1) la comprensibilidad, que significa la medida en que se perciben los eventos de la vida como predecibles, coherentes y estructurados; 2) la manejabilidad, entendida como el grado en que la persona considera que dispone de recursos y capacidades para manejar las situaciones cotidianas, y 3) la significatividad, que es la dimensión motivacional y representa la medida en que se convierte lo que se hace (5) en satisfactorio y con significado para la vida, para ver los acontecimientos adversos como desafíos, que comprometen y orientan positivamente en la vida (6).

Tener un fuerte SC permite a las personas ver la vida como algo comprensible, manejable y con significado, pues les brinda una confianza y habilidad para identificar y utilizar los recursos psicosociales, entre ellos los materiales (dinero, vivienda o alimentación), los cognitivo-emocionales (conocimiento del mundo real o inteligencia), los valorativos-actitudinales (adquisición de estrategias de afrontamiento o manejo efectivo de emociones), las relaciones interpersonales (apoyo social), los biológicos (genética) y los macrosocioculturales (estabilidad cultural, rituales y religión) (7). Gracias a ello, las personas pueden sentirse partícipes en la toma de decisiones para mantener o mejorar su propia salud.

Diferentes disciplinas de la salud, entre ellas enfermería, han mostrado interés por explorar el SC, como un concepto integrado a la teoría salutogénica, propuesta como una teoría de promoción de la salud que hace hincapié en trabajar con las fortalezas, los recursos y las habilidades de las personas para mantener la salud, en lugar de centrarse en la búsqueda de los factores de riesgo y las causas de la enfermedad. Así es como se ha establecido la relación positiva entre un alto SC y una mejor salud física y bienestar mental (8-10), mayor calidad de vida (5), elección de estrategias de afrontamiento (4), automanejo en enfermedades crónicas (11) y adopción de comportamientos saludables (12). Además, se ha evidenciado que un alto SC modera la angustia y los efectos estresantes derivados de la enfermedad, en adultos que cursan condiciones de salud crónicas y agudas (13), entre ellas las enfermedades cardiovasculares, de forma que las personas con un alto SC suelen tener una buena percepción de su propia salud. 
Para medir el concepto de SC, Antonovsky (7) desarrolló una escala denominada Orientación ante la Vida, que consta de 29 ítems, de los cuales 10 evalúan la dimensión de comprensibilidad; 11, la manejabilidad, y 8, la significatividad. Las respuestas incluyen opciones dentro de una escala semántica de 1 a 7 puntos, que indican sentimientos extremos sobre preguntas o enunciados. La puntuación total corresponde a la suma de los valores de cada pregunta, para un mínimo de 29 y un máximo de 203 puntos, cuanto mayor sea la puntuación total, más fuerte será el SC.

Dado que el enfoque disciplinar de enfermería es el cuidado en las experiencias de la salud humana, evaluar el SC es importante, por la integralidad del concepto, que compila aspectos cognitivos, conductuales y motivacionales, de forma que un alto SC otorga confianza y seguridad para identificar y hacer uso de los recursos internos y del entorno inmediato, con el propósito de encontrar soluciones para conseguir afrontar de forma exitosa la enfermedad (14).

El instrumento Orientación ante la Vida ha sido traducido a más de 33 idiomas y ha demostrado adecuadas cualidades métricas (15); sin embargo, y ya que resulta necesario contar con medidas válidas y confiables para su uso en la investigación y la práctica en el contexto y poblaciones específicas, se llevó a cabo la adaptación transcultural y validez facial, validez de contenido, validez de constructo y consistencia interna del cuestionario Orientación ante la Vida de 29 ítems, proceso que se realizó en una población con enfermedad crónica-HTA, dentro de un programa de seguimiento al riesgo cardiovascular, con el fin de disponer de una escala aplicable al contexto local, especialmente para su uso en estrategias orientadas la promoción y el mantenimiento de la salud (16).

\section{Método}

El presente es un estudio metodológico (17), con abordaje cuantitativo. Para la aplicación del cuestionario se utilizó un tipo de muestreo por conveniencia y para la selección del tamaño de muestra se estableció un número entre 5 y 10 participantes por cada ítem que incluye el instrumento (18). Por ello, se utilizó una muestra de 220 personas adultas con HTA que asistieron a una Empresa Social del Estado (ESE) y que cumplieron con los siguientes criterios de inclusión: participantes mayores de 18 años, que tuvieran diagnóstico médico de HTA, inscritos en un programa de riesgo cardiovascular, con más de 6 meses de tratamiento, que supieran leer y que no contaran con algún tipo de discapacidad visual, auditiva o déficit cognitivo. Se excluyeron los pacientes con hipertensión secundaria e hipertensión asociada al embarazo.

\section{Procedimiento}

La investigación se desarrolló en tres fases secuenciales, las cuales correspondieron a fase previa, desarrollo y confirmación (19), que incluyeron el proceso de traducción, adaptación cultural, retrotraducción y validación del instrumento (20). En la fase de desarrollo se obtuvo la autorización para adaptación cultural, validación y uso del instrumento por parte del doctor Avishai Antonovsky, titular de los derechos de autor.

Por su parte, la adaptación cultural siguió los siguientes pasos: 1) traducción independiente desde el idioma inglés al español por parte de dos traductores oficiales; 2) consenso por panel de expertos conformado por investigadoras, traductores y un lingüista, quienes en conjunto evaluaron la equivalencia semántica y cultural entre la versión original y la traducida, producto de lo cual se obtuvo una versión preliminar; 3 ) envío de la versión a tres expertos para la realización de la validez aparente y de contenido, y 4) retrotraducción de esta versión por un tercer traductor oficial, después de la aprobación por parte de los expertos en la validez facial y de contenido, enviándose al titular de los derechos de autor quien la revisó y aprobó. 


\section{Validez facial}

La validez facial se evaluó con 20 adultos y el juicio de tres expertos. Los criterios para evaluar en cada ítem fueron la comprensión (al leer el ítem se entiende lo que se quiere decir), la claridad (frase o ítem con lenguaje y redacción fácil de comprender) y la precisión (frase o ítem puntual y exacto que no da lugar a dudas). Para analizar la concordancia entre los jueces, se utilizó la prueba estadística del coeficiente de kappa de Fleiss, que expresa cualitativamente la fuerza de concordancia entre los evaluadores y cuyo resultado se evaluó con la interpretación dada por Landis y Koch que se consideró aceptable, cuando los índices kappa fueran mayores de 0,41 , lo cual representa una fuerza de concordancia entre expertos moderada (21).

\section{Validez de contenido}

Con respecto a la validez de contenido, se realizó con la participación de tres expertas: una psicóloga con especialización clínica y dos doctoras en enfermería con experiencia en cuidado a personas con enfermedades crónicas y salud mental, quienes también participaron en la validez facial. En la evaluación se tuvo en cuenta el índice de Lawshe modificado por Tristán-López (22), el cual considera un ítem como aceptable cuando reporta un índice de validez de contenido (CVR') de 0,5823 , independiente del número de expertos incluidos en la evaluación. Los criterios para evaluar en cada ítem fueron tres: no necesario, útil pero no esencial y esencial.

\section{Validez de constructo}

Por su parte, la validez de constructo se llevó a cabo por medio de un análisis factorial exploratorio, mediante el análisis de componentes principales con rotación Varimax. Adicionalmente, con el fin de comprobar las dimensiones subyacentes a los ítems, previamente se realizaron las pruebas de Kaiser-Meyer-Olkin (KMO> $0,6)$ y la prueba de esfericidad de Bartlett $(\mathrm{p}<0,05)$, con el propósito de evaluar la idoneidad de los datos para la realización del análisis.

\section{Confiabilidad}

Finalmente, para calcular la consistencia interna del instrumento, se estableció el uso del alfa de Cronbach. Esta prueba estadística representa una medida de homogeneidad y se realizó con las mismas personas que participaron en el proceso de validez de constructo. De esta manera, la consistencia interna, a partir de este cálculo, se considera aceptable si el coeficiente es mayor a 0,70 .

\section{Consideraciones éticas}

Durante el desarrollo de todo el proceso de investigación, se tuvo en cuenta la Resolución 8430 de 1993 del Ministerio de Salud de Colombia (23), la Ley 911 de 2004 (24) y las pautas éticas internacionales para la investigación biomédica con seres humanos (25). Se contó con la aprobación del Comité de Ética de la Facultad de Enfermería de la Universidad Nacional de Colombia y con la autorización de la institución donde se recogió la información. Los participantes del estudio recibieron información sobre su objetivo, y una vez aceptaron la participación voluntaria, firmaron el consentimiento. Para el proceso de traducción, adaptación cultural, validez y confiabilidad desde la consistencia interna, se obtuvo el permiso del titular de los derechos de autor del instrumento. 


\section{Resultados}

\section{Traducción y adaptación cultural}

Para lograr la adaptación transcultural y la equivalencia semántica del instrumento al idioma español, a partir de las observaciones realizadas por los pacientes y expertos, fueron necesarias algunas modificaciones en palabras, sin que esto afectara la estructura y los conceptos que subyacen, como lo corroboró el filial del autor del instrumento. Tal fue el caso del ítem 10, con la palabra consistent, que se traduce al español como consistente, frecuentemente asociada con algo firme o sólido, por lo que fue necesario ajustarla por la palabra consecuente, que refleja la condición de llevar una vida en la que el pensamiento y las acciones coinciden entre sí.

\section{Validez facial}

De los resultados obtenidos en la evaluación realizada por los 20 pacientes, se evidenció una aceptabilidad del $92 \%$, claridad del 90 \% y comprensión del $91 \%$. Sobre los tres aspectos, el índice de kappa de Fleiss mostró un acuerdo moderado a partir de las evaluaciones de los jueces, y en categorías de claridad se obtuvo un valor de 0,50; en precisión, 0,41; y comprensión, 0,49.

\section{Validez de contenido}

El resultado de la prueba estadística arrojó un índice de Lawshe modificado de 0,87 que, de acuerdo con la interpretación propuesta por Tristán-López (22), resulta ser satisfactorio, por lo que se mantuvieron todos los ítems.

\section{Validez constructo}

De las 220 personas que participaron en esta etapa, la mayoría fueron mujeres (72,3\%); el promedio de edad fue de 65,1 años con un rango entre los 50 y los 82 años; el 73,1\% de los pacientes tenía como máximo estudios secundarios; el $62 \%$ tenía estado civil casado o en unión libre, y en cuanto a la ocupación, el $42,3 \%$ se dedicaba a labores del hogar, el resto eran pensionados $(23,6 \%)$, trabajadores independientes $(17,3 \%)$ y empleados $(16,8 \%)$ (tabla 1$)$. 
TABLA 1.

Características sociodemográficas de los 220 pacientes con hipertensión arterial

\begin{tabular}{lr}
\hline Características & Valores \\
\hline Edad; media \pm DE, rango & $65,1 \pm 7,4,50-82$ \\
\hline Sexo; n (\%) & \\
\hline Mujer & $159(72,3)$ \\
\hline Hombre & $61(27,7)$ \\
\hline Nivel de escolaridad; n (\%) & $63(28,6)$ \\
\hline Primaria & $98(44,5)$ \\
\hline Secundaria & $59(26,8)$ \\
\hline Técnico o universitaria & $138(62,7)$ \\
\hline Estado civil; n (\%) & $32(37,3)$ \\
\hline Casado o unión libre & \\
\hline $\begin{array}{l}\text { Soltero, divorciado o } \\
\text { viudo }\end{array}$ & $93(42,3)$ \\
\hline Ocupación; n (\%) & $52(23,6)$ \\
\hline Hogar & $38(17,3)$ \\
\hline Pensionado & $37(16,8)$ \\
\hline Trabajador independiente
\end{tabular}

Fuente: elaboración propia

Previo al análisis factorial, tanto el valor de $\mathrm{KMO}=0,790$ como la significación estadística de la prueba de esfericidad de Bartlett $(\chi 2=1691,88 ; \mathrm{p}<0,01)$ confirmaron que los datos eran apropiados para el análisis factorial exploratorio. Al realizar el análisis factorial, utilizando como método de estimación el de componentes principales y la rotación Varimax, este último con el propósito de facilitar la interpretación de los datos, se obtuvieron los resultados contenidos en la tabla 2.

TABLA 2.

Carga factorial de los ítems del instrumento tras rotación Varimax

\begin{tabular}{|c|c|c|c|}
\hline Ítem & F1 & F2 & F3 \\
\hline 1. Cuando le habla a la gente, ¿siente que no le entienden? & 0,4897 & 0,0748 & 0,3732 \\
\hline $\begin{array}{l}\text { 2. En el pasado, cuando tuvo que realizar algo que dependiera de la cooperación de otras personas, } \\
\text { usted sintió quc... }\end{array}$ & 0,2067 & 0,4020 & 0,0485 \\
\hline $\begin{array}{l}\text { 3. Excluyendo a las personas más cercanas a usted, ¿qué tan bien conoce a la mayor parte de la gente } \\
\text { con la que está en contacto diariamente? }\end{array}$ & 0,0289 & 0,4696 & 0,0209 \\
\hline 4. ¿Tiene la sensación de que en realidad no le importa lo que pasa a su alrededor? & 0,5297 & 0,0824 & 0,1743 \\
\hline 5. ¿Le ha sorprendido en el pasado el comportamiento de gente que pensaba conocer bien? & 0,1330 & 0,1727 & 0,6677 \\
\hline 6. ¿Sc ha sentido defraudado por personas con las que contaba? & 0,0996 & 0,4113 & 0,5656 \\
\hline 7. La vida es... & 0,5140 & 0,0979 & 0,1170 \\
\hline 8. Hasta ahora su vida... & 0,2675 & 0,1814 & 0,3949 \\
\hline 9. ¿Tiene la sensación de que se le está tratando de forma injusta? & 0,0084 & 0,5866 & 0,2045 \\
\hline 10. En los últimos 10 años, su vida... & 0,2104 & 0,4981 & 0,0947 \\
\hline 11. La mayor parte de las cosas que haga en el futuro, probablemente será... & 0,6098 & 0,1321 & 0,0364 \\
\hline 12. ¿Tiene la sensación de que está en una situación incierta y no sabe qué hacer? & 0,2608 & 0,6368 & 0,0751 \\
\hline 13. Quées lo que mejor describe su forma de ver la vida? & 0,6781 & 0,0600 & 0,1587 \\
\hline 14. Cuando piensa sobre su vida, con frecuencia... & 0,5997 & 0,1137 & 0,3745 \\
\hline 15. Cuando se enfrenta un problema difícil, elegir una solución es... & 0,1508 & 0,4591 & 0,1113 \\
\hline 16. Hacer las cosas que hace todos los días es... & 0,6846 & 0,1457 & 0,0225 \\
\hline 17. Su vida cn d futuro, probablementc... & 0,5418 & 0,3241 & 0,1699 \\
\hline 18. Cuando en el pasado le ha sucedido algo desagradable, su reacción ha sido... & 0,3328 & 0,1334 & 0,4074 \\
\hline 19 ¿Tiene sentimientos $\mathrm{e}$ ideas muy confusas? & 0,1464 & 0,7199 & 0,0663 \\
\hline 20. Cuando realiza algo que lo hace sentirse bien, es cierto que... & 0,4387 & 0,2894 & 0,0340 \\
\hline $21 .{ }^{\mathrm{H} H a}$ tenido sentimientos que hubiera preferido no sentir? & 0,1989 & 0,5883 & 0,2600 \\
\hline 22. Puede prever que su vida personal en el futuro... & 0,5096 & 0,3723 & 0,3322 \\
\hline 23. ¿Picnsa que simpre habrá gente con la que pucda contar cn cl futuro? & 0,5717 & 0,1206 & 0,2028 \\
\hline 24. ¿Tiene usted la sensación de no saber exactamente qué le va a pasar? & 0,0814 & 0,4583 & 0,1571 \\
\hline $\begin{array}{l}\text { 25. Mucha gente, aun con carácter fuerte, a veces se siente triste o fracasada en ciertas situaciones. } \\
\text { ¿Con qué frecuencia se ha sentido así en el pasado? }\end{array}$ & 0,3826 & 0,3794 & 0,5931 \\
\hline 26. Cuando algo ha ocurrido, usted generalmente ha encontrado que... & 0,0300 & 0,0682 & 0,4933 \\
\hline $\begin{array}{l}\text { 27. Cuando picnsa en las dificultades importantes que probablcmentc cnfrentará cn su vida, usted } \\
\text { siente que... }\end{array}$ & 0,6523 & 0,0711 & 0,2192 \\
\hline 28. ¿Con qué frecuencia siente que las cosas que hace en su vida tienen poco significado? & 0,4160 & 0,3312 & 0,1068 \\
\hline 29. ¿Con qué frecuencia siente que no está seguro de poder mant & 0,2216 & 0,0042 & 0,5334 \\
\hline
\end{tabular}

Fuente: elaboración propia

El análisis factorial exploratorio muestra un reajuste de los ítems que integrarían cada una de las tres dimensiones definidas originalmente por el autor. De esta manera, los factores quedaron conformados de la siguiente manera: el primero agrupó los ítems 1, 4, 7, 11, 13, 14, 16, 17, 20, 22, 23, 27 y 28, y fue básicamente equivalente al componente de significatividad propuesto por Antonovsky, dado que incluyó siete ítems (4, 7, $11,14,16,22$ y 28 ) de los ocho que representan esta dimensión. Sin embargo, al inspeccionar el contenido, se identificó que este factor refleja lo que las personas perciben frente a las cosas que hacen en su vida y el significado que le asignan a la ayuda que reciben de los demás, e incluyó además ítems pertenecientes por 
construcción a las dimensiones de comprensibilidad con los ítems ( 1 y 17) y manejabilidad con los ítems (13, 20,23 y 27), que mostraron aspectos relacionados con las expectativas y optimismo frente al presente y futuro. Esta dimensión se comprende como la evaluación que hace el individuo de su propia vida.

El segundo factor quedó conformado por los ítems 2, 3, 9, 10, 12, 15, 19, 21 y 24, agrupando siete de los once ítems que representan la comprensibilidad e incluye los ítems 2 y 9 , que originalmente hacen parte de la dimensión de manejabilidad y que se relacionan con algún grado de previsibilidad y planeación ante posibles cambios en el futuro, sumado a una sensación de confianza frente a las acciones y ayuda recibida por parte de otras personas.

El tercer factor quedó formado por los ítems 5, 6, 8, 18, 25, 26 y 29, relacionado de forma distante con la dimensión de manejabilidad, ya que coincidió con cuatro ítems $(6,18,25$ y 29) de los diez definidos por el autor, lo que refleja aspectos relacionados con retrospección de la vida.

Teniendo en cuenta lo anterior, se encontró que aun cuando la conformación de los factores resultantes de la solución del análisis factorial exploratorio se aproxima a lo descrito por el autor, esto no corresponden exactamente con los ítems diseñados para medir cada componente. Al estimar el número de factores, se encontró que el $36,21 \%$ de la varianza fue explicada por tres factores: el primero de ellos representó la mayor parte de la varianza $(20,60 \%)$ y abarcó 13 ítems de la escala; mientras que los otros dos factores representaron un porcentaje adicional de la varianza explicada, del 15,61\%.

\section{Confiabilidad}

El alfa de Cronbach reportó un valor $\alpha=0,82$ para el total de la escala, lo que indica que es lo suficientemente confiable como para usarla.

\section{Discusión}

Este estudio describe el proceso de adaptación cultural y validación del cuestionario Orientación ante la Vida, que mide el SC. Conceptualmente, se refiere a una capacidad general de percibir experiencias de vida que le permitan actuar de forma constructiva en situaciones desfavorables. El SC ha demostrado ser útil para evaluar el afrontamiento por parte de las personas con enfermedad cardiovascular; especialmente, la literatura habla sobre la relación entre un fuerte SC y comportamientos saludables (26), salud física y mental (27) y manejo de los síntomas (28).

Ahora, teniendo en cuenta que al utilizar instrumentos desarrollados en otros países e idiomas es necesario la adaptación cultural, en este estudio se siguió un proceso basado en las guías internacionales, con el fin de superar la traducción literal de un idioma a otro, de modo que se evite el riesgo de llevar a cabo interpretaciones erróneas. Por ello, se garantizó que la redacción de los ítems se diera en términos idóneos para la cultura y los usos habituales del español en el contexto colombiano. Además, fueron necesarios cambios en algunas palabras, de modo que fueran coherentes con el contexto y con la cultura, sin que ello afectara el sentido de las preguntas, ni los conceptos que subyacen a la versión original, como lo corroboró el titular de los derechos de autor.

El proceso de validez facial realizado mediante el kappa de Fleiss, que refleja la concordancia interobservador, dejó ver un acuerdo moderado en los aspectos de comprensión, claridad y precisión, al superar el rango de aceptable que para el presente estudio fue considerado el mínimo para definir qué ítems requerirían una nueva revisión en términos de redacción, con el fin de afinar la semántica buscando un mayor acuerdo entre los jueces. Y como se mencionó en la fase de adaptación transcultural, el instrumento tuvo modificaciones, las cuales no afectaron la estructura del instrumento original y lo que se pretende medir, hecho que fue corroborado a través de las pruebas de validez y fiabilidad. 
Adicional a lo anterior, los resultados del análisis factorial exploratorio mostraron la agrupación de los ítems en tres factores que dan cuenta del 36,21 \% de la varianza explicada. Los resultados obtenidos están conforme a lo descrito por Cea (29), quien considera que un $30 \%$ de varianza explicada es un valor adecuado en las escalas del área social.

El mismo Antonovsky propuso que el SC estaba compuesto por dominios teóricamente bien definidos: comprensibilidad, manejabilidad, significatividad, pese a que existe cierta controversia en la literatura sobre la unidimensionalidad del constructo. En este sentido, varios estudios (30-34) han analizado la estructura interna del cuestionario mediante el análisis exploratorio y han llegado a proponer cuatro estructuras factoriales, por ser las que más apoyo empírico tienen.

Así, la primera estructura corresponde a un factor global denominado SC, sugerida por el autor del instrumento (30,31). La segunda estructura corresponde a tres factores en los que se abordan los tres componentes del SC de forma diferenciada, pero con altas correlaciones entre sí. La tercera estructura representa dos factores, el primero de ellos integra los ítems de los componentes de manejabilidad y significatividad y el segundo corresponde al componente de comprensibilidad. La cuarta y última estructura es un factor de segundo orden con tres componentes, con la concepción del SC como un constructo unidimensional (32).

Los resultados del presente estudio, en relación con la estructura interna del instrumento, apuntan a la conformación de los elementos de la escala cargados en tres componentes, coincidiendo con otros estudios $(33,34)$ que evidenciaron hallazgos similares. En este sentido, los resultados se suman a la evidencia a favor de la solución multifactorial del constructo, planteamiento que puede estar relacionado con que el autor es explícito en aseverar el carácter teórico de la escala, por lo que difícilmente mantiene congruencia con los modelos factoriales.

Por otra parte, el análisis factorial ayuda a aclarar el significado de los componentes del SC. En este estudio, el primer factor parece ser el más destacado, ya que explicó la mayor parte de la varianza; sin embargo, como lo refieren Nunnally y Bernstein (35) obtener varios factores no significa que no se esté midiendo un único rasgo bien definido y con consistencia conceptual. Así, las saturaciones de los ítems que se agruparon tienen similitud con el componente que representa la significatividad y reflejan aspectos asociados con los sentimientos de optimismo hacia la vida, hallazgos que se asemejan a lo encontrado en otros análisis factoriales (36). El segundo factor debe considerarse esencialmente como la validación del componente de comprensibilidad; mientras que el tercer factor no tiene una conexión fuerte con ninguno de los componentes de Antonovsky, aunque existe una relación más cercana con la manejabilidad que con los otros dos componentes.

$\mathrm{Al}$ ser esta una primera exploración de la estructura interna del cuestionario en el contexto colombiano, se propone continuar aplicándolo en diferentes poblaciones, de forma que se demuestre congruencia o similitud con la solución factorial reportada. Por otra parte, es posible contrastar estadísticamente en futuras investigaciones la hipótesis basada en la agrupación de los ítems y corroborar si la estructura empírica de la escala coincide con la teórica mediante un análisis factorial confirmatorio, ya que la literatura disponible no propone una estructura factorial consistente para someter a confirmación.

Para terminar, en este estudio la consistencia interna de la escala, estimada mediante el alfa de Cronbach, fue de 0,82 , y resulta adecuada máxime si se compara con el obtenido en un estudio realizado en Brasil en pacientes con enfermedad coronaria, donde dicho coeficiente fue de 0,78 (37). Esta cifra se encuentra dentro de los rangos reportados en una revisión sistemática que tuvo por objetivo analizar la validez y la fiabilidad de los instrumentos que medían el SC y en cuyos resultados se encontró que los alfa de Cronbach oscilaban entre 0,70 y $0,95(15)$.

La evaluación de la validez y la confiabilidad de instrumentos que miden constructos subjetivos relacionados con la salud, por parte de los profesionales de enfermería, refleja el interés de la disciplina por disponer de herramientas apropiadas para utilizar en la práctica clínica y la investigación en situaciones 
particulares, por lo que resulta fundamental conocer el modelo conceptual que respalda la construcción teórica del instrumento y su función (38). En esta línea, una teoría que se está consolidando en el área de la promoción de la salud, que hace posible ver las acciones en salud orientadas a alcanzar o mantener un mayor control sobre la salud, el bienestar y el envejecimiento saludable, es la teoría salutogénica que incorpora el SC como un recurso que se correlaciona positivamente con los comportamientos saludables y que influye en el afrontamiento de la enfermedad. De ahí que su evaluación permitirá diseñar intervenciones individualizadas que estimulen el desarrollo de habilidades para mejorar la salud (39) en pacientes con enfermedades crónicas como la HTA.

Entre las limitaciones del estudio está que la muestra se obtuvo de una institución urbana y en adultos sin tener en cuenta otras poblaciones, como la de los adolescentes; además, el muestreo no probabilístico supone una limitación para generalizar los resultados.

\section{Conclusiones}

En este artículo se evidencia que el instrumento Orientación ante la Vida mantiene la equivalencia semántica y conceptual con la versión original, y podría usarse en población similar para evaluar el concepto de SC en pacientes con HTA. Se ofreció un análisis sobre la estructura factorial del instrumento y se confirmó su multidimensionalidad con la presencia de tres factores; sin embargo, se plantea la necesidad de realizar otros estudios, a fin de proponer una estructura factorial consistente, además de someter el instrumento a un análisis factorial confirmatorio, de modo que se pueda explicar el comportamiento observado en la agrupación de los ítems que conforman cada dimensión.

Por lo tanto, el estudio contribuye a la generación de un instrumento adaptado al idioma español y población adulta del municipio de Ibagué (Tolima, Colombia) y se convierte en un punto de partida para medir el concepto de SC en adultos con HTA, para realizar intervenciones e investigaciones que contribuyan a mejorar las estrategias de afrontamiento de los pacientes.

\section{Referencias}

1. Rosendorff C, Lackland DT, Allison M, Aronow WS, Black HR, Blumenthal RS, et al. Treatment of hypertension in patients with coronary artery disease: a scientific statement from the American Heart Asociation, American College of Cardiology, and American Society of Hypertension. Hypertension. 2015;65:1372-407.

2. Sarki AM, Nduka CU, Stranges S, Kandala NB, Uthman OA. Prevalence of hypertension in low-and middle-income countries: A systematic review and meta-analysis. Medicine (Baltimore). 2015;94(50):1-16. https://doi.org/10 $.1097 / \mathrm{MD} .0000000000001959$

3. Orozco-Gómez ÁM, Castiblanco-Orozco L. Factores psicosociales e intervención psicológica en enfermedades crónicas no transmisibles. Rev Colomb Psicol. 2014;24(1):203-17. https://doi.org/10.15446/rcp.v24n 1.42949

4. Kristofferzon ML, Engström M, Nilsson A. Coping mediates the relationship between sense of coherence and mental quality of life in patients with chronic illness: a cross-sectional study. Qual Life Res. 2018;27(7):1855-63. http ://dx.doi.org/10.1007/s11136-018-1845-0

5. Silarova B, Nagyova I, Rosenberger J, Van Dijk JP, Reijneveld SA. Sense of coherence as a mediator between hostility and health-related quality of life among coronary heart disease patients. Hear Lung. 2016;45(2):126-31. http:/ /dx.doi.org/10.1016/j.hrtlng.2015.11.004

6. Super S, Wagemakers M, Picavet H, Verkooijen K, Koelen M. Strengthening sense of coherence: opportunities for theory building in health promotion. Health Promot Int [Internet]. 2016;31:869-78. Disponible en: https://ac ademic.oup.com/heapro/article-lookup/doi/10.1093/heapro/dav071

7. Antonovsky A. Unraveling the mystery of health: how people manage stress and stay well. San Francisco: Wiley; 1987. 
8. Izydorczyk B, Sitnik-Warchulska K, Kühn-Dymecka A, Lizińczyk S. Resilience, sense of coherence, and coping with stress as predictors of psychological well-being in the course of schizophrenia: the study design. Int J Environ Res Public Health. 2019;16(7):1266. https://doi.org/10.3390/ijerph16071266

9. Langeland E, Forbech $\mathrm{H}$. The application of salutogenesis in mental healthcare settings. En: Mittelmark M, Sagy S, Eriksson M, Bauer G, Pelikan J, Lindström B, editores. The handbook of salutogenesis. New York: Springer; 2017. p. 299-304.

10. Del-Pino-Casado R, Espinosa-Medina A, López-Martínez C, Orgeta V. Sense of coherence, burden and mental health in caregiving: a systematic review and meta-analysis. J Affect Disord. 2019;242(1):14-21. https://doi.or $\mathrm{g} / 10.1016 /$ j.jad.2018.08.002

11. Li Z, Liu T, Han J, Li T, Zhu Q, Wang A. Confrontation as a mediator between sense of coherence and selfmanagement behaviors among elderly patients with coronary heart disease in North China. Asian Nurs Res (Korean SC Nurs Sci). 2017;11(3):201-6. https://doi.org/10.1016/j.anr.2017.08.003

12. Nilsen V, Bakke PS, Rohde G, Gallefoss F. Is sense of coherence a predictor of lifestyle changes in subjects at risk for type 2 diabetes? Public Health. 2015;129:155-61. http://dx.doi.org/10.1016/j.puhe.2014.12.014

13. Winger JG, Adams RN, Mosher CE. Relations of meaning in life and sense of coherence to distress in cancer patients: a meta-analysis. Psychooncology. 2016;25(1):2-10. https://doi.org/10.1002/pon.3798

14. Juvinya-Canal D. Salutogénesis, nuevas perspectivas para promover la salud. Enferm Clín. 2013;23(3):87-8. http s://doi.org/10.15446/rcp.v24n1.42949

15. Eriksson M, Lindström B. Validity of Antonovsky's sense of coherence scale: a systematic review. J Epidemiol Community Health. 2005;59(6):460-6. https://doi.org/10.1136/jech.2003.018085

16. Sangster-Gormley E, Frisch N, Schreiber R. Articulating new outcomes of nurse practitioner practice.J Am AsSC Nurse Pract. 2013;25(12):653-8. https://doi.org/10.1002/2327-6924.12040

17. LoBiondo-Wood G, Judith H. Nursing research methods and critical appraisal for evidence-based practice. 8th ed. St. Louis, Missouri: Elsevier; 2014.

18. Norman G, Streiner D. Bioestadística. Madrid: Mosby/Doyma; 1996.

19. Muñiz J, Elosua P. Directrices para la traducción y adaptación de los tests: segunda edición. Psicothema. 2013;25(2):151-7. https://doi.org/10.7334/psicothema2013.24

20. Ramada Rodilla JM, Serra Pujadas C, Clanchet Delclós LG. Adaptación cultural y validación de cuestionarios de salud: revisión y recomendaciones metodológicas. Salud Pública Méx [internet]. 2013;55(1):57-66. Disponible en: http://www.scielo.org.mx/scielo.php?script=sci_arttext\&pid=S0036-36342013000100009

21. Landis RJ, Koch GG. The measurement of observer agreement for categorical data. Biometrics. 1977;33(1):159-74. https://doi.org/10.2307/2529310

22. Tristán-López A. Modificación al modelo de Lawshe para el dictamen cuantitativo de la validez de contenido de un instrumento objetivo. Av Medición [internet]. 2003;6(1):37-48. Disponible en: http://www.humanas.unal. edu.co/psicometria/files/8413/8574/6036/Articulo4_Indice_de_validez_de_contenido_37-48.pdf

23. Ministerio de Salud de la República de Colombia. Resolución 008430/1993, por la cual se establecen las normas científicas, técnicas y administrativas para la investigación en salud [internet]. Bogotá. Disponible en: https://w ww.minsalud.gov.co/sites/rid/Lists/BibliotecaDigital/RIDE/DE/DIJ/RESOLUCION-8430-DE-1993.PDF

24. República de Colombia. Ley 911 de 2004, por la cual se dictan disposiciones en materia de responsabilidad deontológica para el ejercicio de la profesional de enfermería en Colombia [internet]. Disponible en: http://ww w.mineducacion.gov.co/1621/articles-105034_archivo_pdf.pdf

25. Consejo de Organizaciones Internacionales de las Ciencias Médicas. Pautas éticas para la investigación biomédica en seres humanos preparadas por el Consejo de Organizaciones Internacionales de las Ciencias Médicas (CIOMS). Ginebra; 2002.

26. Silarova B, Nagyova I, Rosenberger J, Studencan M, Ondusova D, Reijneveld SA, et al. Sense of coherence as a predictor of health-related behaviours among patients with coronary heart disease. Eur J Cardiovasc Nurs. 2014;13(4):345-356. https://doi.org/10.1177/1474515113497136 
27. Kocjan J. Sense of coherence as a predictor of physical and psychoSCial health among cardiac patients. J Educ Heal Sport. 2015;5(6):241-50. http://dx.doi.org/10.5281/zenodo.18558

28. Falk Kristin SK. Fatigue is a prevalent and severe symptom associated with uncertainty and sense of coherence in patients with chronic heart failure. Eur J Cardiovasc Nurs. 2007;6:99-104. https://doi.org/10.1016/j.ejcnurse .2006 .05 .004

29. Cea D’Ancona MÁ. Análisis multivariante teoría y práctica en la investigación social. 2. ed. Madrid: Sintesis; 2002.

30. Antonovsky A. The structure and properties of the sense of coherence scale. Soc Sci Med. 1993;36(6):725-33.

31. Söderhamn U, Sundsli K, Cliffordson C, Dale, B. Psychometric properties of Antonovsky's 29-item sense of coherence scale in research on older home-dwelling Norwegians. Scand J Public Health. 2015;43(8):867-74. h ttps://doi.org/10.1177/1403494815598863

32. Rivera de los Santos FJ, López AM, Ramos P, Moreno M del C. Propiedades psicométricas de la escala sentido de coherencia (SC-29) en adolescentes españoles. Child Adolesc Psychol [internet]. 2011;4:11-39. Disponible en: http://hdl.handle.net/11441/41008

33. Von Humboldt $S$, Leal I. The Orientation to Life Questionnaire: validation of a measure to assess older adults' sense of coherence. Educ Gerontol. 2015;41(6):451-65. https://doi.org/10.1080/03601277.2014.983373

34. Bachem R, Maercker A. Development and psychometric evaluation of a revised sense of coherence scale. Eur J Psychol Assess. 2016;34(3):206-15. https://doi.org/10.1027/1015-5759/a000323

35. Nunnally JC, Bernstein IH. Psychometric theory. 3r ed. New York: McGraw-Hill; 1994.

36. Bengtsson-Tops A, Brunt D, Rask M. The structure of Antonovsky's sense of coherence in patients with schizophrenia and its relationship to psychopathology. Scand J Caring Sci. 2005;19(3):280-7. https://doi.org/ $10.1111 / \mathrm{j} .1471-6712.2005 .00342 . x$

37. Spadoti Dantas RA, Silva FSE, Ciol MA. Psychometric properties of the Brazilian Portuguese versions of the 29and 13-item scales of the Antonovsky's Sense of Coherence (SC-29 and SC-13) evaluated in Brazilian cardiac patients. J Clin Nurs. 2014;23(1-2):156-65. https://doi.org/10.1111/jocn.12157

38. Echevarría-Guanilo ME, Gonçalves N, Romanoski PJ. Psychometric properties of measurement instruments: conceptual bases and evaluation methods-Part I. Texto Context Enferm [Internet]. 2017;26(4). Disponible en: http://www.revenf.bvs.br/scielo.php?script=sci_arttext\&pid=S0104-07072017000400326

39. Bauer GF, Roy M, Bakibinga P, Contu P, Downe S, Eriksson M, et al. Future directions for the concept of salutogenesis: a position article. Health Promot Int. 2019;1-9.

\section{Notas}

* Artículo original de investigación

Conflictos de interés: las autoras declaran no tener conflictos de interés.

\section{Licencia Creative Commons CC BY 4.0}

Cómo citar este artículo: Esquivel Garzón N, Díaz Heredia LP. Validez y confiabilidad del cuestionario Orientación ante la Vida de Antonovsky (SC-29 ítems), versión en español. Investig Enferm Imagen Desarr. 2020;22. https://doi.org/10.11144/Javeriana.ie22.vcco 